Research Paper

\title{
Examining the Effect of Teleconferences on Oncology Phase I Trials
}

\author{
Alexandra Mckane ${ }^{1}$, Chao Sima², Sharon Fleck ${ }^{1}$, Glen J. Weiss ${ }^{1,2,}$, \\ 1. Virginia G. Piper Cancer Center Clinical Trials at Scottsdale Healthcare, Scottsdale, AZ, USA; \\ 2. The Translational Genomics Research Institute, Phoenix, AZ, USA.
}

$\triangle$ Corresponding author: Glen J. Weiss, M.D. Cancer Treatment Centers of America, 14200 W. Celebrate Life Way, Goodyear, AZ 85338. Phone: 623-207-3000 Fax: 623-207-3003 Email: Glen.Weiss@ctca-hope.com.

( ) Ivyspring International Publisher. This is an open-access article distributed under the terms of the Creative Commons License (http://creativecommons.org/ licenses/by-nc-nd/3.0/). Reproduction is permitted for personal, noncommercial use, provided that the article is in whole, unmodified, and properly cited.

Received: 2013.05.06; Accepted: 2013.06.26; Published: 2013.07.04

\begin{abstract}
Objective: Phase I clinical trials are the first stage of clinical development of an investigational agent. Because the trials often take place at several geographically dispersed sites, safety teleconferences are held to update investigators and the drug sponsor on safety information and other pertinent business related to the trial conduct. Here we examine associations between the frequency of teleconferences and other clinical trial factors on trial conduct efficiency.

Methods: We examined Phase I clinical trials for patients with solid tumors opened for enrollment at a single, non-profit cancer center in Arizona (Center) that had completed at least three dose levels. The following information was included: safety teleconference frequency, whether or not the sponsor or contract research organization sent follow-up requests for updates on patient accrual, and safety outside of scheduled safety teleconferences. The dose escalation scheme, route of study drug administration and formulation type (e.g. oral targeted therapy or monoclonal antibody) was also included.

Results: Forty-nine Phase I studies were examined for inclusion. The majority of safety teleconferences were regularly scheduled (81.6\%) with most taking place bi-weekly $(46.9 \%)$. Additional solicitation for updates outside of scheduled safety teleconferences were requested during the conduct of $3 \mathrm{I}(63.3 \%)$ studies. None of the factors analyzed were significantly associated with accrual, subject dosing, and dose escalation.

Conclusion: We found that the frequency of teleconferences does not appear to expedite phase I study accrual, subject dosing, or dose escalation in the first 3 cohorts of a phase I clinical trial.
\end{abstract}

Key words: Phase 1 clinical trials, teleconferences

\section{INTRODUCTION}

A phase 1 clinical trial is the first stage of clinical development of an investigational agent towards evaluating safety and toxicity and to determine the recommended phase 2 dose [1]. With the typical phase 1 trial geographically dispersed at multiple sites, safety teleconferences are held to update investigators and the study sponsor on potential drug safety issues, dose escalation scheduling, and other pertinent business regarding the conduct of the trial.
Study sponsors may hire a contract research organization (CRO) or organize their own in-house study administrators to oversee study accrual and cohort dose escalation at the study site(s) [2]. Although direction has been given on promoting the productivity of teleconferences [3], there has been no published research on how the timing and frequency of these safety teleconferences may affect consenting, dosing, and dose escalation. Here we examine interactions 
between the frequency of teleconferences with the date of consenting, dosing, and cohort escalation; and their effect on trial conduct efficiency.

\section{METHODS}

\section{Data Acquisition}

All information was collected after local institutional review board approval with a data cutoff date of July 31, 2012. We included in this study only Phase 1 clinical trials for patients with solid tumors opened for enrollment between January 2006 and July 2012 at a single, non-profit cancer center in Arizona (Center) that had completed at least three dose levels. The following information was examined: safety teleconference frequency and whether or not the sponsor/CRO send follow-up requests for updates on patient accrual and safety outside of scheduled safety teleconferences. Because of various study designs and other potential factors that might interfere with dose escalation [1], data collection was limited to only the first three dose levels from each study. This included: the opening date for the first three dose levels, date the first subject on each cohort consented, date the first subject dosed on each cohort, and if there was a dose-limiting toxicity (DLT) on a given cohort. Information about whether the study sponsor hired a CRO or kept study administration inhouse was included. The dose escalation schema, route of study drug administration and formulation type (injectable targeted therapy, oral targeted therapy, oral and injectable targeted therapy, cytotoxic therapy, or monoclonal antibody) was also included. A sponsor was designated as either a "small cap" or "large cap" firm based on its market capitalization (below or above $\$ 1.5$ billion; respectively, as of July 2012). Phase 2 or 3 studies were excluded from this analysis. Studies lacking a recorded date of the first safety teleconference and/or those that did not have any patients enrolled on the first cohort at Center were censored for this information but included in the overall analysis.

\section{Statistical Evaluation}

For each study, days between cohorts opening and first patient consenting were computed for all three dose escalation cohorts and then averaged (Consenting - Opening). The same calculation was done between cohorts opening and first patient dosing (Dosing - Opening) and the first patient consenting and the first patient dosing (Dosing - Consenting). Additionally, days between the first cohort opening and the first teleconference (when available) was computed ( $1^{\text {st }}$ Teleconference $-1^{\text {st }}$ Opening). Association analyses of these timelines with different factors (listed in Table 2), as well as, first-in-human (FIH) and
non-FIH studies were carried out using Kruskal Wallis test.

\section{RESULTS}

Forty-nine Phase 1 studies were examined for inclusion. We were not able to procure the date of the first safety teleconference for eight out of the 49 included studies. Characteristics of the study collected are listed in Table 1 . The most common type of study drug formulation was an injectable targeted therapy $(46.9 \%)$ or was administered orally $(36.7 \%)$. Forty-one of the studies were FIH trials $(83.7 \%)$. Most of the study sponsors were companies with a market value of less than $\$ 1.5$ billion, or "small cap" (65.3\%). The majority of safety teleconferences were regularly scheduled $(81.6 \%)$ with most taking place bi-weekly $(46.9 \%)$ and only nine $(18.4 \%)$ teleconferences were conducted irregularly. Of these nine studies, three of the sponsors did not send any requests for patient updates.

A majority of the administrators made requests for patient safety and enrollment updates outside of teleconferences via email (63.3\%). A majority $(61.2 \%)$ of the study sponsors contracted with outside CROs to conduct and/or monitor the clinical trial. The dose escalation schema used consisted mainly of the modified Fibonacci " $3+3$ " sequence $(67.3 \%)$, followed by "accelerated titration" (24.5\%), then finally "other" $(8.2 \%)$.

We analyzed the effect of time of cohort opening to patient consent, time of cohort opening to subject dosing, and time of consent to subject dosing and to see if any of these measures were significantly associated with the frequency of safety teleconferences, dose escalation schema, route of study drug administration, use of a CRO, or sponsor's size (market capitalization). For all comparisons, none of these factors had a significant association (Kruskal Wallis test; all p-values > 0.05) (Table 2). We did not find any statistically significant differences between FIH studies and non-FIH studies (data not shown). There were too few DLT events $(n=7$ with each one occurring in a separate study) within the cohort dose escalations to incorporate into our analyses across these 49 studies.

\section{DISCUSSION}

There are no formal guidelines or data that we are aware of that define clinical trial conduct efficiency. We posit that if there are potentially measurable items that can be identified and prolong the time to subject consent, subject dosing, and cohort dose escalation. Prolongation of these timeframes can have impact on the drug development process. Safety teleconferences are a common way for a clinical trial study administrator to communicate with researchers 
at a clinical trials site and instantaneously to share relevant information with each other. However, with the digital age offering other alternatives such as email, we wonder if there may be even more efficient ways to communicate rapidly when required.

Table I. Characteristics of Included Studies (N=49).

\begin{tabular}{ll}
\hline Method of Administration & Number of Studies (n=49) \\
Injectable targeted therapy & $23(46.9 \%)$ \\
Oral targeted therapy & $18(36.7 \%)$ \\
Monoclonal Antibody & $4(8.2 \%)$ \\
Cytotoxic therapy & $3(6.1 \%)$ \\
Oral and injectable targeted therapy & $1(2.1 \%)$ \\
Sponsor Size by Market capitalization & Number of Studies (n=49) \\
Small** & $32(65.3 \%)$ \\
Large* & $17(34.7 \%)$ \\
Frequency of Teleconferences & Number of Studies (n=49) \\
Bi-weekly & $23(46.9 \%)$ \\
Weekly & $17(34.7 \%)$ \\
Irregular & $9(18.4 \%)$ \\
Requests outside of teleconferences & Number of Studies (n=49) \\
Requests made & $31(63.3 \%)$ \\
No requests made & $18(36.7 \%)^{* * *}$ \\
Drug Administration Modality & Number of Studies (n=49) \\
CRO (Trial administrated by a contract research organization) & $30(61.2 \%)$ \\
In-house (Trial administrated by the sponsor) & $19(38.8 \%)$ \\
Dose Escalation Schema & Number of Studies (n=49) \\
$3+3$ (modified Fibonacci) & $33(67.3 \%)$ \\
Accelerated titration & $12(24.5 \%)$ \\
Other & $4(8.2 \%)$ \\
\hline Key: Large &
\end{tabular}

Key: ${ }^{*}$ Large $=$ market capitalization $>\$ 1.5$ billion USD as of July 2012; **Small $=$ market capitalization $\leq \$ 1.5$ billion USD as of July 2012; "Irregular" $=$ safety

teleconference calls were $>4$ weeks apart from one another. ${ }^{* * *} 3$ out of these 18 studies had irregularly scheduled calls.

Table 2. Analyses for Time against Key Events.

\begin{tabular}{|c|c|}
\hline Effect of Time of $1^{\text {st }}$ Safety Teleconference To First Cohort Opening $(\mathrm{N}=41)$ & p-value \\
\hline Dose Escalation Schema & 0.07 \\
\hline Drug Administration Modality & 0.42 \\
\hline Teleconference Frequency & 0.88 \\
\hline Whether or not a CRO was used & 0.98 \\
\hline Study sponsor size & 0.65 \\
\hline Requests outside of teleconferences & 0.96 \\
\hline \multicolumn{2}{|l|}{ Effect of Time of Cohort Opening to Consent $(\mathrm{N}=49)$} \\
\hline Dose Escalation Schema & 0.14 \\
\hline Drug Administration Modality & 0.57 \\
\hline Teleconference Frequency & 0.95 \\
\hline Whether or not a CRO was used & 0.59 \\
\hline Study sponsor size & 0.83 \\
\hline Requests outside of teleconferences & 0.33 \\
\hline \multicolumn{2}{|l|}{ Effect of Time of Cohort Opening to Subject Dosing $(N=49)$} \\
\hline Dose Escalation Schema & 0.06 \\
\hline Drug Administration Modality & 0.66 \\
\hline Teleconference Frequency & 0.83 \\
\hline Whether or not a CRO was used & 0.84 \\
\hline Study sponsor size & 0.49 \\
\hline Requests outside of teleconferences & 0.34 \\
\hline \multicolumn{2}{|l|}{ Effect of Time of Consent to Subject Dosing ( $N=49)$} \\
\hline Dose Escalation Schema & 0.68 \\
\hline Drug Administration Modality & 0.54 \\
\hline Teleconference Frequency & 0.68 \\
\hline Whether or not a CRO was used & 0.60 \\
\hline Study sponsor size & 0.25 \\
\hline Requests outside of teleconferences & 0.55 \\
\hline
\end{tabular}


Our data suggests that weekly and bi-weekly scheduled teleconferences are likely excessive and unnecessary for oncology phase 1 clinical trial conduct efficiency. Furthermore, the use of CROs for trial administration did not influence any of the factors analyzed. Besides the regularly scheduled safety teleconferences, additional information was often solicited by the study sponsor in the interval between teleconferences. Consequently, it can be concluded that any questions, concerns, or safety issues that may arise during the conduct of the study could be disseminated via email without impediment to successful patient enrollment, patient consent, and dose escalation on a clinical trial. Based on our data, we would suggest ad hoc teleconference meetings should a safety issue arise and not schedule regular meetings, while maintaining frequent, as needed communication by email to report adverse events and potential accrual issues. This can be of further value as globalization of studies becomes more common and significant differences in time zones adds to safety teleconferencing scheduling constraints.

Our analysis and conclusions may be limited by the small sample size. Additionally, clinical trial cohort opening dates was limited to information available at the Center. Approximately 75\% of the 25 first-in-human single-agent phase I trials (1992-2008) for anticancer agents that were eventually approved by the Food and Drug Administration (FDA) for the treatment of solid tumors exceeded 4 dose levels [4]. Because DLTs are rare events in the initial dose escalation of oncology phase 1 clinical trials [4] we were not able to get a statistically significant sample size of DLTs to analyze in relation to teleconferences and cohort expansion timing. In addition, meaningful assessment of the role of regularly vs. irregularly scheduled safety teleconferences to ensure safety cannot be answered. In this study, we found that the frequency of teleconferences does not appear to expedite phase 1 study accrual, subject dosing, or dose escalation in the first three cohorts of a phase 1 clinical trial. To our knowledge there are no pharmaceutical industry or CRO data addressing how the frequency of teleconferences may affect phase 1 clinical trial efficiency. An economic analysis on the time and wages for staffing at the CRO, study site(s), and sponsor should be considered to further evaluate the cost contribution of inefficient safety teleconferences on drug development.

\section{ACKNOWLEDGMENTS}

We thank the patients, clinical trial team, and data managers for their contributions and assistance in procuring teleconference data.

\section{COMPETING INTERESTS}

The authors have declared that no competing interest exists.

\section{REFERENCES}

1. Caldwell S, Sima C, Jameson G, Fleck S, Weiss GJ. Factors Influencing Time to Determination of the Recommended Phase 2 Dose in Phase 1 Clinical Trials. Am J Clin Oncol 2013; 36:146-50.

2. Goldenberg NA, Spyropoulos AC, Halperin JL, et al. Improving academic leadership and oversight in large industry-sponsored clinical trials: the ARO-CRO model. Blood. 2011; 117:2089-92.

3. [No authors listed]. Follow These Steps to a Productive Teleconference: Assign Specific Tasks, Outline Agenda, and Highlight Objectives to Avoid Confusion. Urology Times . 2001 Nov;: 38.

4. Le Tourneau C, Lee JJ, Siu LL. Dose escalation methods in phase I cancer clinical trials. J Natl Cancer Inst. 2009; 101:708-20. 